\title{
Essai d'interprétation de la répartition des Ascaridoidea chez les Mammifères
}

\author{
par A. J. PETTER \\ Laboratoire de Zoologie (Vers), associé au C.N.R.S. \\ Muséum national d'Histoire naturelle, 43, rue Cuvier, F 75231 Paris Cedex 05.
}

\section{Résumé.}

Un schéma évolutif est proposé pour expliquer la répartition des Ascarides chez les Mammifères et les Oiseaux, basé sur la notion de périodes géologiques d'expansion pour les lignées parasitaires. On suppose que la période d'expansion des Ascarides chez les Mammifères s'est produite ultérieurement à la diversification des groupes de Mammifères primitifs, ce qui explique pourquoi ces derniers sont dépourvus d'Ascarides. Les périodes d'expansion ont dû coïncider avec celle des Carnivores et des Siréniens, qui ont été envahis les premiers, puis avec celles des Artiodactyles et des Equidés, qui sont parasités par des genres plus évolués. Les autres cas de parasitisme des Mammifères par les Ascarides sont interprétés comme des phénomènes de capture.

\section{Summary.}

\section{Evolutionary aspects of the distribution of Ascaridoidea in Mammals.}

In an attempt to interpret the distribution of Ascaridoidea in Mammals and Birds, a scheme is proposed, based on the concept of geological periods of evolutionary expansion for parasitic lines.

It is assumed that the evolutionary expansion period of Ascaridoidea in Mammals occurred after the diversification of primitive mammals; this would explain the absence of ascarids in these groups. Periods of evolution appear to coincide with the diversifcation of Carnivora and Sirenia, the first to become invaded; Artiodactyla and Equidae became invaded later, at the time of their diversification. The other cases of parasitism of Mammals by Ascarids are thought to be "captures".

Accepté le 12 novembre 1976. 
Les travaux de Osche (1958) ont mis en évidence que la super-famille des Ascaridoidea obéit aux lois de la spécificité phylogénique, c'est-à-dire que l'on y observe une évolution parallèle entre l'hôte et le parasite. Osche a, d'autre part, montré l'importance chez les Ascarides des phénomènes de capture. Rappelons que la notion de capture définit le passage d'un parasite déjà différencié à un hôte nouveau, auquel il s'adapte par de simples spéciations (voir Osche, 1957, «Wirtskreiserweiterung »). Osche oppose ainsi les parasites «autochtones " qui ont évolué en même temps que leurs hôtes, aux parasites «allochtones» qui sont le résultat d'une capture.

Cependant, Inglis (1965) a fait remarquer que l'évolution parallèle entre hôte et parasite ne s'observe qu'à une échelle zoologique très large. En effet, si le parallélisme entre l'évolution des différentes familles d'Ascarides et celles des classes de leurs hôtes est parfait, il ne s'observe plus si l'on étudie la distribution des genres chez les Mammifères (tableau I). Ces genres sont en effet répartis dans des ordres d'hôtes variés et n'appartenant pas à la même lignée. Depuis les travaux de Osche, une nouvelle notion est apparue dans les travaux récents concernant l'évolution des Nématodes, la notion d'époque géologique d'expansion pour les parasites (Chabaud, Bain et Puylaert, 1966; Durette-Desset, 1971; Quentin, 1971; Chabaud, 1971). Suivant cette notion, plutôt que les affinités zoologiques, ce sont les périodes géologiques d'apparition des hôtes qui sont déterminantes dans la distribution d'une lignée parasitaire; les lignées parasitaires présentent en effet des périodes où elles sont aptes à évoluer, suivies de périodes où elles n'évoluent plus; à la période où ils évoluent, les parasites vont envahir tous les hôtes qui s'épanouissent au même moment, et offrent donc un grand nombre de niches écologiques disponibles. Dans cette nouvelle conception, le terme de parasites « autochtones » est réservé aux parasites qui se sont adaptés à leurs hôtes à la période géologique où ceux-ci se sont diversifiés, et le terme de parasites «allochtones 》 ou parasites de capture à ceux qui se sont adaptés à leurs hôtes à n'importe quelle époque géologique, ultérieurement à la diversification de ceux-ci.

Nous allons essayer d'interpréter la répartition des Ascarides chez les Mammifères, exposée dans le tableau I, en nous appuyant sur cette nouvelle conception.

Nous avons suivi dans ce tableau la classification donnée par Hartwich (1974), sauf en ce qui concerne le genre Lagochilascaris.

Pour les spectres d'hôtes, nous avons consulté les travaux de Sprent (1957, 1959, 1968, 1970, 1972, 1973), Warren (1970, $1971 a$, et $b, 1972)$, Mozgovoi, Kornienko et Shakhamatova (1969), Maung (1975).

Deux faits, déjà observés par Osche, caractérisent cette répartition : d'une part, les Mammifères primitifs sont dépourvus d'Ascarides adultes, d'autre part, les Ascarides sont principalement parasites des Carnivores, des Artiodactyles et des Périssodactyles, c'est-à-dire des principaux ordres de la cohorte des Ferungulata de Simpson (1945). Les genres les plus primitifs se trouvent chez les Carnivores et les plus évolués chez les Artiodactyles et les Equidés. Osche expliquait cette répartition par l'hétéroxénie du cycle biologique des Ascarides primitifs, ayant pour conséquence que 
Tableau I. - Répartition des Ascarides chez les Mammifères et les Oiseaux

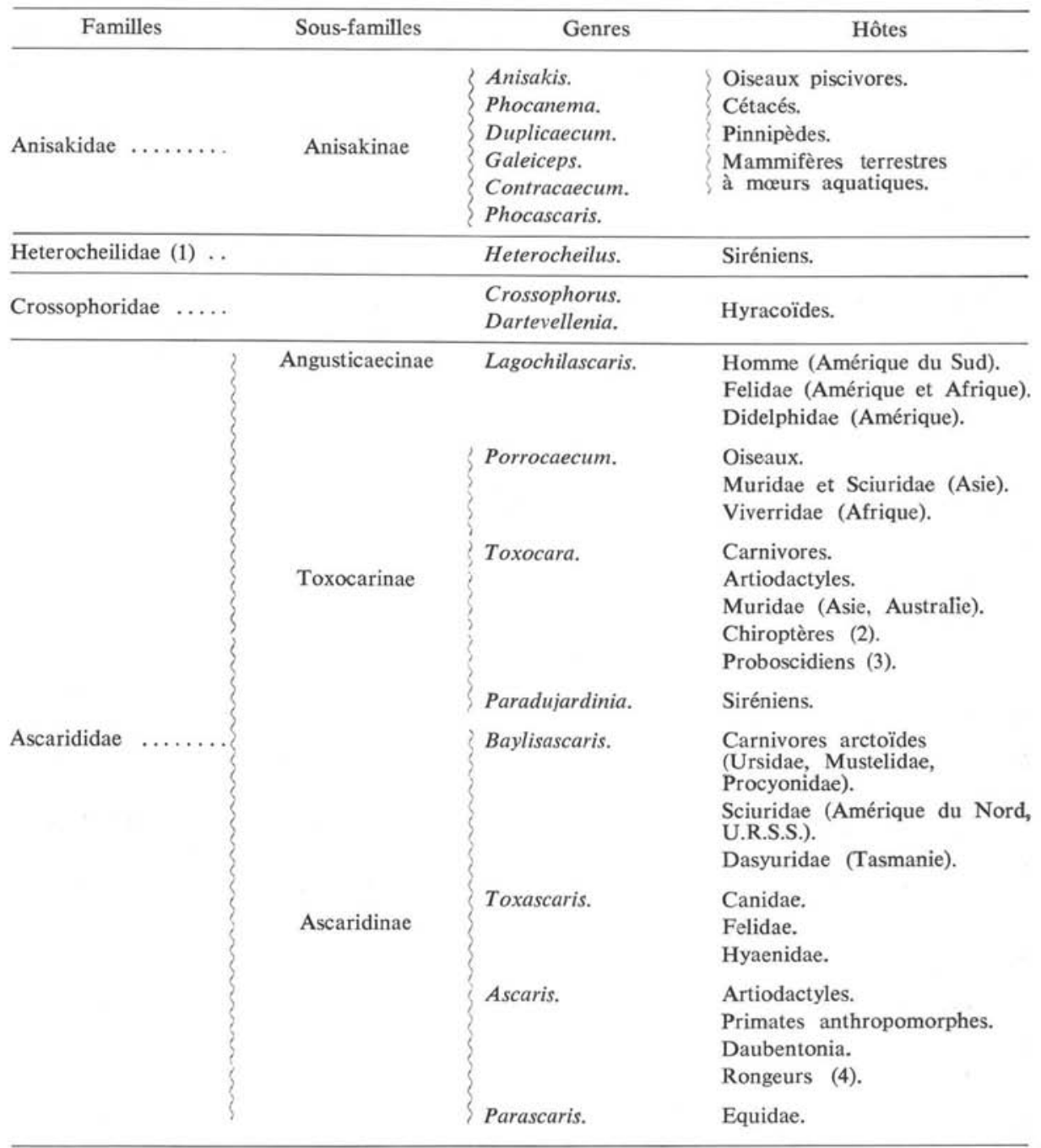

(1) D'après Chabaud et Bain (1965), la famille des Heterocheilidae, mal conmue, pourra vraisemblablement disparaître quand les affinités des deux espèces qui la composent auront été établies.

(2) Des Ascarides ont été signalés deux fois chez les Chiroptères: Toxocara cynonycteridis (Parona, 1889), parasite de Xantharpyia amplexicaudata, mal connu, n'a pas été revu depuis sa description originale. Toxocara pteropodis est signalé par Baylis $(1936 a)$ chez un Pteropodidé aux Nouvelles Hébrides. Warren (1971), reprenant le matériel de Baylis a montré qu'il s'agissait réellement d'une espèce distincte du genre Toxocara, mais comme elle n'a été trouvée qu'une fois, on ne peut affirmer avec certitude qu'elle soit réellement un parasite de Pteropodidae, et non pas un parasite accidentel.

(3) Des Ascarides ont été signalés seulement deux fois chez les Eléphants, uniquement chez des animaux en captivité, et les spécimens sont insuffisamment décrits pour être attribués avec certitude au genre Toxocara (Baylis, 1936 b).

(4) La validité ou l'appartenance au genre Ascaris des espèces parasites de Rongeurs est discutée (voir Tiner, 1951; Sprent, 1957, 1973). 
seuls les Carnivores aient pu se contaminer, les Herbivores ne pouvant l'être qu'après l'établissement de la monoxénie.

Cependant, cette hypothèse n'explique pas que des Mammifères plus anciens que les Carnivores, comme les Marsupiaux, les Insectivores, les Chiroptères, qui ont également un régime insectivore ou franchement carnivore, soient dépourvus d'Ascarides.

En fait, tous les Mammifères reliques, Monotrèmes (1), Marsupiaux, Dermoptères, Insectivores, Chiroptères (2), Tubulidentés, Xénarthres (3), Pholidotes, Prosimiens, qui paraissent avoir peu évolué depuis le début de l'éocène, sont indemnes d'Ascarides quel que soit leur régime (nous verrons plus loin que les quelques cas de parasitisme par les Ascarides connus chez ces groupes, généralement limités à une famille d'hôtes et à une région, sont interprétés comme des phénomènes de capture).

Nous supposons donc que la période d'expansion des Ascarides chez les Mammifères a dû se produire un peu plus tardivement, au moment de l'explosion évolutive des Ferungulata, et nous proposons le schéma évolutif suivant (fig. 1).

\section{Parasites autochtones.}

La première adaptation des Ascarides aux Vertébrés supérieurs s'est effectuée chez les Oiseaux, donnant naissance au genre Porrocaecum.

Ultérieurement, une nouvelle phase d'adaptation correspond à l'expansion des Carnivores et des Siréniens, avec les genres Toxocara et Paradujardinia, puis Baylisascaris et Toxascaris; enfin, les genres Ascaris et Parascaris s'individualisent au moment de l'expansion des Artiodactyles et des Equidés.

L'isolement de la famille des Crossophoridae s'explique aisément par l'isolement de leurs hôtes Hyracoïdes sur le continent africain (ils ont dû se différencier sur ce continent à partir des Angusticaecinae de Reptiles et donner une lignée séparée).

\section{Parasites allochtones.}

Tous les autres cas de parasitisme par les Ascarides chez les Mammifères sont interprétés comme des phénomènes de capture.

Nous ne reviendrons pas sur le cas des Anisakinae, exposé par Osche (1958), qui sont des parasites de capture issus des Anisakidae parasites de Poissons.

D'après Durette $(1963,1964)$ et Sprent (1971), le genre Lagochilascaris est vraisemblablement également un parasite de capture issu d'Ascarides de Vertébrés inférieurs, et appartient à la famille des Angusticaecinae parasites de Reptiles.

Les autres cas de capture se sont effectués à une échelle zoologique moins importante, les hôtes d'origine étant déjà des Vertébrés supérieurs.

(1) Ophidascaris radiosa (Schneider, 1866), Baylis, 1921, décrit par Schneider chez un Echidné et attribué par Baylis aux Vipères, est vraisemblablement un parasite accidentel (voir Osche, 1958).

(2) En ce qui concerne les Ascarides de Chiroptères, voir note 2, p. 44.

(3) Deux espèces ont été signalées chez les Xénarthres: Ascaris heringii (Linstow, 1879) est insuffisamment décrit pour être attribué à un genre précis. Ascaris dasypodina Baylis, 19222, présente d'après Sprent (1968) des affinités avec Lagochilascaris; il s'agirait dans ce cas d'un parasite de capture. 


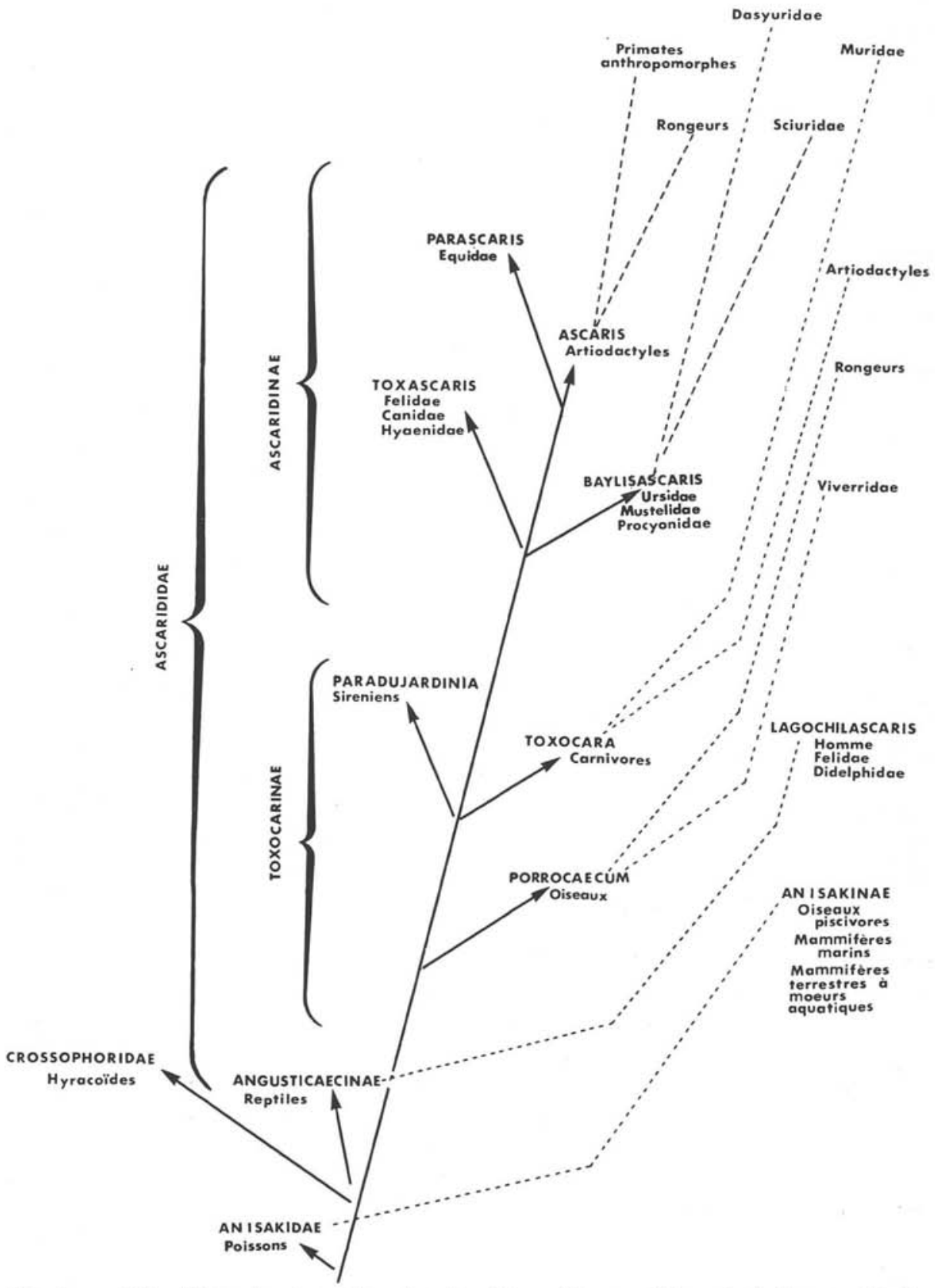

Fig. 1. - Répartition des Ascarides chez les Mammifères: schéma évolutif proposé. Les hôtes autochtones sont figurés à gauche, distribués le long de l'arbre principal, et les hôtes allochtones à droite, à l'extrémité des lignes en tirets. 
- Cas des Ascarides de Rongeurs.

Osche a fait remarquer que les Ascarides de Rongeurs sont vraisemblablement des parasites allochtones. Ils en présentent en effet les caractéristiques: les Rongeurs sont dans l'ensemble peu parasités, les espèces parasites sont réparties dans différents genres fondamentalement parasites d'autres ordres de Mammifères; de plus, dans les familles parasitées, le parasitisme est souvent localisé géographiquement (voir Sprent, 1957, 1973). Au point de vue biologique, l'existence chez Toxocara mackerrasae d'un cycle monoxène très raccourci, s'opposant aux cycles des autres Toxocara (voir Warren, 1970), est également pour cette espèce un argument en faveur d'une capture.

\section{- Cas des Toxocara d'Artiodactyles.}

Durette-Desset et Chabaud (1974) expliquent la présence de deux espèces du genre primitif Toxocara chez les Artiodactyles d'apparition paléontologique récente, par une capture qui se serait produite en Afrique à partir des parasites de Carnivores: leur hypothèse est appuyée sur des arguments morphologiques; les Toxocara d'Artiodactyles et de Rongeurs présentent des caractères particuliers correspondant à l'ancien genre Neoascaris, qui, par la structure des lèvres, marque une évolution par rapport aux Toxocara.

- Cas des Ascaris de Primates.

On sait que l'Ascaris lumbricoides parasite de l'Homme, est à peine distinguable morphologiquement de l'Ascaris suum des porcs; il est donc facile d'admettre que cette espèce est le résultat d'une capture assez récente des Ascarides d'Artiodactyles.

D'après Sprent (1968), l'Ascaris petiti, parasite du lémurien malgache Daubentonia, ne différerait pas réellement d'Ascaris lumbricoides et serait donc vraisemblablement un parasite accidentel. S'il s'agissait vraiment d'une espèce distincte, son existence s'expliquerait par une capture au second degré effectuée après l'arrivée de l'Homme à Madagascar.

- Cas du Baylisascaris de Marsupiaux.

L'extraordinaire cas de parasitisme des Marsupiaux Dasyuridae tasmaniens par une espèce du genre Baylisascaris (voir Sprent, 1970 ; Sprent, Lamina et MacKeown, 1973) est plus difficile à expliquer.

Sprent envisage plusieurs hypothèses, mais conclut plutôt en faveur d'un parasitisme ancestral apporté par les Marsupiaux au moment de leur arrivée dans la région australienne. Cependant, bien que la date de cette arrivée soit mal connue, elle est sûrement antérieure à l'apparition des Carnivores arctoïdes, qui sont parasités par Baylisascaris; de plus, il semble improbable que dans ce cas, le parasitisme soit resté limité au seul groupe des Dasyuridae et à une seule petite région de Tasmanie.

Sprent pense qu'il ne peut s'agir d'une capture effectuée dans la région australienne, puisque les Carnivores placentaires n'y sont jamais parvenus, à l'exception du 
Dingo, introduit tardivement par l'Homme et par ailleurs dépourvu de Baylisascaris; l'hypothèse d'une capture à partir d'animaux importés encore plus récemment en tant qu'animaux familiers, de cirques ou de jardins zoologiques, lui semble très improbable car une spéciation aussi importante que celle qui différencie l'espèce B. tasmaniensis n'aurait pas eu le temps de se produire. Cependant, c'est malgré tout cette dernière hypothèse qui nous semble la plus vraisemblable, car elle expliquerait la répartition géographique très étroite du parasitisme, et l'on sait que les. phénomènes de capture sont particulièrement faciles dans les îles et que, d'autre part, les phénomènes de spéciation se montrent particulièrement rapides chez des. hôtes très différents de l'hôte originel.

\section{Bibliographie}

Baylis (H. A.), $1936 a$. - A new ascarid from a bat. Ann. Mag. nat. Hist., 10, 360-365.

Baylis (H. A.), 1936 b. - The fauna of British India, including Ceylon and Burma. 1. Nematoda. Taylor and Francis, édit., London, 408 p.

Chabaud (A. G.), 1971, - Evolution of host-parasite adaptation in nematodes of Vertebrates. Int. J. Parasit., 1, 217-221.

Сhabaud (A.-G.) et Bain (O.), 1965. - Description de Hartwichia rousseloti n. gen., n. sp., ascaride parasite de Crocodile et remarques sur la famille des Heterocheilidae Railliet et Henry, 1912. Bull. Mus. Nat. Hist. Nat., Sér. 2, 37, 848-853.

Chabaud (A.-G.), Bain (O.) et Puylaert (F.), 1967. - Description de trois nouveaux Nématodes Molineinae et considérations sur la systématique et le caractère archaïque de cette sous-famille. Bull. Mus. Nat. Hist. Nat., Sér. 2, 38, $904-$ 920.

DurktTE (M.-C.), 1963. - Remarques sur les anomalies du genre Lagochilascaris. Bull. Soc. Path. Exot., 56, 129-133.

Durette (M.-C.), 1964. - Nématodes parasites de Mammifères et de Reptiles. 4. Phasmidiens ( $2^{\circ}$ note). Mission G.-F. de Witte, Parc National de l'Upemba, 63, 3-12.

Durette-Desset (M.-C.), 1971. - Essai de classification des Nématodes Héligmosomes. Corrélations avec la paléobiogéographie des hôtes. Mém. Mus. Nat. Hist. Nat., sér. A, Zool., 69, 1-126.

Durette-Desset (M.-C.) et Chabaud (A.-G.), 1974. - Trois nouveaux Nématodes parasites du Chevrotain aquatique: Hyemoschus aquaticus au Gabon (collection G. Dubost). Bull. Mus. Nat. Hist. Nat., Sér. 3, Zool., 135, 75-87.

Hartwich (G.), 1974. - Keys to genera of the Ascaridoidea. CIH Keys to the nematode parasites of vertebrates. Commonwealth Agricultural Bureaux, Farnham Royal, Bucks, England, No. 2, 15 p.

INGLIS (W. G.), 1965. - Patterns of evolution in parasitic Nematodes. In: A. E. R. Taylor. Evolution of Parasites. Third Symposium of the British Society for Parasitology, pp. 79-124. Blackwell Scientific Publications, édit., Oxford. 
MaUng (M.), 1975. - An ascaridoid nematode from a hippopotamus. Int. J. Parasit., 5, 431-439.

Mozgovoi (A. A.), Kornienko (Z.P.) et Shakhmatova (V. I.), 1969. - Ascaris skrjabini n. sp. (Ascaridata) a parasite of the camel. Parazitologiya, 3, 174-175.

Osche (G.), 1957. - Die «Wirtskreiserweiterung 》 bei parasitischen Nematoden und die sie bedingenden biologisch -ökologischen Faktoren. Z. ParasitKde, 17, 437-489.

Osche (G.). 1958. - Beiträge zur Morphologie, Ökologie und Phylogenie der Ascarıdoidea (Nematoda). Parallelen der Evolution von Parasit und Wirt. Z. ParasitKde, $18,479-572$.

QueNtin (J.-C.), 1971. - Sur les modalités d'évolution chez quelques lignées d'Helminthes de Rongeurs Muroidea. Cah. O.R.S.T.O.M., Sér. Ent. Méd. Parasitol., 9, 103-176.

Simpson (G. G.), 1945. - The principles of classification and a classification of mammals. Bull. Amer. Mus. Nat. Hist., 85, 1-350.

SPRENT (J.F. A.), 1957. - A new species of Neoascaris from Rattus assimilis with a redefinition of the genus. Parasitology, 47, 350-360.

SPRENT (J.F.A.), 1959. - The life history and development of Toxascaris leonina (von Linstow, 1902) in the dog and cat. Parasitology, 49, 330-373.

Sprent (J.F.A.), 1968. - Notes on Ascaris and Toxascaris, with a definition of Baylisascaris gen. nov. Parasitology, 58, 185-198.

SPRENT (J.F. A.), 1970. - Baylisascaris tasmaniensis sp. nov. in marsupial carnivores: heirlom or souvenir? Parasitology, 61, 75-86.

SPRENT (J.F. A.), 1971. - Speciation and development in the genus Lagochilascaris. Parasitology, 62, 71-112.

SPRENT (J.F. A.), 1972. - Toxocara vajrasthirae sp. nov. from the hog-badger (Arctonyx collaris) of Thailand. Parasitology, 65, 491-498.

SPRENT (J.F. A.), 1973. - The ascaridoid nematodes of rodents with a redescription of Porrocaecum ratti. Parasitology, 66, 367-380.

Sprent (J.F. A.), Lamina (J.) et Mackeown (A.), 1973. - Observations on migratory behaviour and development of Baylisascaris tasmaniensis. Parasitology, 67, 67-83.

TINeR (J.D.), 1951. - The morphology of Ascaris laevis Leidy, 1856, and notes on ascarids in rodents. Proc. Helminth. Soc. Wash., 18, 126-131.

Warren (E. G.), 1970 a. - Observations on the life-cycle of Toxocara mackerrasae. Parasitology, 60, 239-253.

Warren (E. G.), 1970 b. - Ascaris species in warthogs. Ann. Parasitol. hum. comp., 45, 251-252.

Warren (E. G.), 1971 a. - A new species of Toxascaris from hyenas. Parasitology, 62, 171-178.

WARREN (E. G.), $1971 b$. - Studies on the morphology and taxonomy of the genera Toxocara Stiles, 1905 and Neoascaris Travassos, 1927. Zool. Anz., 185, 393-442.

Warren (E. G.), 1972. - Two new species of Toxocara from viverrid hosts. Parasitology, $65,179-187$. 